\title{
Pharmacokinetic/Pharmacodynamic Considerations of Alternate Dosing Strategies of Tocilizumab in COVID-19
}

\author{
Elizabeth Leung ${ }^{1,2,3}$ (1) Ryan L. Crass ${ }^{4}(1) \cdot$ Sarah C. J. Jorgensen ${ }^{5,6} \cdot$ Sumit Raybardhan $^{7} \cdot$ Bradley J. Langford $^{8}$. \\ W. Justin Moore ${ }^{9}$ Nathaniel J. Rhodes ${ }^{9,10,11}$ (B)
}

Accepted: 9 November 2021 / Published online: 11 December 2021

(c) The Author(s), under exclusive licence to Springer Nature Switzerland AG 2021

\begin{abstract}
Tocilizumab is one of few treatments that have been shown to improve mortality in patients with coronavirus disease 2019 (COVID-19), but increased demand has led to relative global shortages. Recently, it has been suggested that lower doses, or fixed doses, of tocilizumab could be a potential solution to conserve the limited global supply while conferring equivalent therapeutic benefit to the dosing regimens studied in major trials. The relationship between tocilizumab dose, exposure, and response in COVID-19 has not been adequately characterized. There are a number of pharmacokinetic (PK) parameters that likely differ between patients with severe COVID-19 and patients in whom tocilizumab was studied during the US FDA approval process. Likewise, it is unclear whether a threshold exposure is necessary for tocilizumab efficacy. The safety and efficacy of fixed versus weight-based dosing of tocilizumab has been evaluated outside of COVID-19, but it is uncertain if these observations are generalizable to severe or critical COVID-19. In the current review, we consider the potential advantages and limitations of alternative tocilizumab dosing strategies. Leveraging PK models and simulation analyses, we demonstrate that a fixed single dose of tocilizumab $400 \mathrm{mg}$ is unlikely to produce PK exposures equivalent to those achieved in the REMAP-CAP trial, although weight-stratified dosing appears to produce more uniform exposure distribution. Data from current and future trials could provide PK/pharmacodynamic insight to better inform dosing strategies at the bedside. Ultimately, rational dosing strategies that balance available limited supply with patient needs are required.
\end{abstract}

\section{Introduction}

Tocilizumab, a monoclonal antibody that inhibits interleukin (IL)-6 signaling by binding to soluble and membranebound IL-6 receptors (IL-6R), has been shown to improve mortality in patients with severe or critical coronavirus disease 2019 (COVID-19) [1, 2]. It is currently recommended

Elizabeth Leung

beth.leung@utoronto.caAuthors and Affiliations

1 Department of Pharmacy, St. Michael's Hospital/Unity Health Toronto, Toronto, ON, Canada

2 Leslie Dan Faculty of Pharmacy, University of Toronto, Toronto, ON, Canada

3 Li Ka Shing Knowledge Institute, Toronto, ON, Canada

4 Ann Arbor Pharmacometrics Group, Ann Arbor, MI, USA

5 Department of Pharmacy, Mount Sinai Hospital, Toronto, ON, Canada

6 Institute of Medical Science, University of Toronto, Toronto, ON, Canada by a number of international, national, and local consensus guidelines for the treatment of severely and critically ill COVID-19 patients, driven largely by the results of two major platform randomized controlled trials in COVID-19 $[3,4]$. Tocilizumab dosing strategies in these trials included banded, weight-based dosing (e.g., $400 \mathrm{mg}$ if $41-65 \mathrm{~kg}$, $600 \mathrm{mg}$ if $66-90 \mathrm{~kg}, 800 \mathrm{mg}$ if $>90 \mathrm{~kg}$ ), and weight-based

7 Department of Pharmacy, North York General, Toronto, ON, Canada

8 Public Health Ontario, Toronto, ON, Canada

9 Department of Pharmacy, Northwestern Medicine, Chicago, IL, USA

10 Department of Pharmacy Practice, College of Pharmacy Downers Grove Campus, Midwestern University, Downers Grove, IL, USA

11 Pharmacometrics Center of Excellence, College of Pharmacy Downers Grove Campus, Midwestern University, Downers Grove, IL, USA 


\section{Key Points}

Studies demonstrating a clinical benefit of tocilizumab in specific subsets of patients with coronavirus disease 2019 (COVID-19) used dosing regimens extrapolated from other approved indications for the drug. Herein, we review pharmacokinetic and pharmacodynamic (PK/ PD) data from tocilizumab across indications to inform rational posology in severe COVID-19.

Current population PK models for tocilizumab suggest that exposure increases non-linearly with increasing body size. Exposure matching to predicted exposures from REMAP-CAP suggests that an alternate weightbanded strategy may provide sufficient drug exposure for the treatment of severe COVID-19; however clinical validation is required.

Medication supply is generally not considered when determining dosing for evaluation in clinical trials, which can pose an issue when medication supply fluctuates unpredictably or is extremely costly, especially during a global pandemic. Future studies, especially those using flexible adaptive platform methodology, should incorporate rational dosing strategies and collect relevant PK/PD data to ensure 'socially optimal' dosing of therapeutics.

dosing (e.g., $8 \mathrm{mg} / \mathrm{kg}$ up to $800 \mathrm{mg}$ ) [3-7]. However, in the absence of exposure-response data to guide dose selection for patients with COVID-19, the optimal tocilizumab dosing strategy in this population remains unclear.

Recently, increased demand coupled with limited production capacity has led to a relative global tocilizumab shortage [8]. Various jurisdictions have had to balance high demand with limited supply [9-12]. Unfortunately, this has disproportionately impacted areas of the world already struggling with a large burden of cases. In light of the current tocilizumab shortage, some jurisdictions have implemented lower, fixed doses of tocilizumab as a rationing strategy with the hope that this will not compromise the observed benefit $[8-10,13]$.

In this article, we review the pharmacokinetics (PK) and pharmacodynamics (PD) of tocilizumab and enumerate potential advantages and limitations of a fixed-dose approach for the treatment of COVID-19. We consider direct clinical experience as well as extrapolated evidence in other patient populations and conditions. We also consider whether fixed dosing is likely to produce less interpatient variability in exposures across the body-weight distribution of adult patients compared with weight-based dosing. Finally, we present the results of model-based simulations to evaluate whether alternative dosing strategies for tocilizumab are likely to yield PK exposures that are substantially similar to those achieved in clinical trials conducted in COVID-19 patients.

\subsection{Overview of Tocilizumab Pharmacokinetics (PK)}

The relationship between tocilizumab dose, exposure, and response in COVID-19 has not been adequately characterized. Weight-based dosing of tocilizumab in COVID-19 was adopted empirically based on experience in treating patients with rheumatological diseases and cytokine release syndrome (CRS) associated with chimeric antigen receptor T-cell (CAR T-cell) therapy. However, the PK properties of monoclonal antibodies leave open the possibility that fixed dosing may reduce interpatient variability in exposure. Such an approach could hypothetically facilitate the use of fixed doses without compromising efficacy or safety.

Due to their large size and hydrophilicity, monoclonal antibodies reside almost exclusively within plasma and extracellular fluid [14], and these fluid compartments frequently do not scale linearly with incremental increases in total body weight [15-18]. Monoclonal antibodies are eliminated via two pathways. First, after administration, they undergo proteolytic catabolism, which is associated with linear clearance (CL). The second pathway involves target engagement (e.g., IL-6R and tocilizumab) on both soluble and membrane-bound targets, followed by internalization and intracellular degradation. This process is complex and is associated with non-linear CL [14] and depends on the relative expression of the target (e.g., IL-6R, sIL-6R). Therefore, this mechanism can be influenced by patient-specific factors such as disease type and severity [14, 19, 20]. Notably, the linear portion of tocilizumab CL appears to scale with body weight [14], but this relationship is imperfect $[18,21]$.

As a result, weight-based dosing of monoclonal antibodies, which linearly proportionalizes the dose to total body weight (as opposed to weight-banded or allometric dose scaling), may result in relative underexposure in low bodyweight patients and relative overexposure in obese patients compared with patients with mid-range body weights [18, 22]. In order to balance the need for appropriate dosing in severe COVID-19, the limited supply of tocilizumab available in some areas, and the potential risk of over- or underexposure within different body-weight strata, a formal comparison of weight-based and alternative dosing strategies is needed.

\subsection{Current Knowledge of Tocilizumab Exposure- Response for Efficacy}

There are no PK/PD targets for tocilizumab with respect to its use in chronic or acute inflammatory conditions 
(e.g., rheumatoid arthritis [RA], CRS) or for patients with COVID-19. Without a defined target concentration (e.g., maximum concentration $\left[C_{\max }\right]$ ) or exposure (e.g., area under the curve $[\mathrm{AUC}]$ ), it is challenging to design diseasespecific, let alone patient-specific, dosing regimens. To date, it remains unclear whether an exposure threshold exists for tocilizumab in order to confer the benefits observed in randomized trials conducted in patients with COVID-19. As a result, individualized response monitoring (i.e., trending) of clinical response, and cautious interpretation of easily obtainable biomarkers (e.g., IL-6 and C-reactive protein [CRP]), has become the norm in the clinical setting. The only exposure-response analysis reported to date is provided in a study by Moes et al. [23]. The investigators predicted individual tocilizumab exposure in 29 patients with severe COVID-19 and computed mean (standard deviation [SD]) exposure based on CRP relapse status (post-dose increase in CRP) and survival. They found no differences in exposure between patients with or without CRP relapse or survivors and non-survivors. Tocilizumab exposure was $>1 \mu \mathrm{g} / \mathrm{mL}$ for at least 15 days in all patients studied. These results must be considered exploratory given the limited sample size and single-dose administration (i.e., all patients received an 8 $\mathrm{mg} / \mathrm{kg}$ dose).

\subsection{Current Knowledge of Tocilizumab Exposure- Response for Safety}

Tocilizumab globally inhibits IL-6R (including both membrane-bound and soluble forms), causing inhibition of potential protective effects of IL-6 and resulting in decreased serum concentrations of acute-phase proteins, including CRP $[13,24]$. While this anti-inflammatory effect can be beneficial in the setting of chronic inflammatory conditions such as RA, global IL-6 inhibition carries the risk of inhibiting an appropriate response to acute infection as well as cell growth and differentiation. Accordingly, adverse events associated with tocilizumab include bacterial infection, intestinal perforation, and pancreatitis [25].

No definitive exposure-toxicity relationship exists for tocilizumab. In the initial US FDA review, patients receiving tocilizumab who weighed $>100 \mathrm{~kg}$ were more likely to experience any infection (41-50\%) and serious infections (1.4-4.5\%) compared with those who weighed $<60 \mathrm{~kg}$ (32-38\% and $1.3-2.7 \%$, respectively). However, the trend towards greater adverse events (including infections) in the $>100 \mathrm{~kg}$ group was similar between patients who received tocilizumab plus a disease-modifying antirheumatic drug (DMARD) compared with those who received a DMARD plus placebo [21]. In healthy volunteers, the highest safe and tolerable dose of tocilizumab that did not adversely impact neutrophil counts was $20 \mathrm{mg} / \mathrm{kg}$ [26]. The highest $C_{\max }$ associated with the maximum tolerated dose $(28 \mathrm{mg} / \mathrm{kg})$ in healthy volunteers was $649 \mu \mathrm{g} / \mathrm{mL}$ [26], which is higher than would typically be experienced with a single $8 \mathrm{mg} / \mathrm{kg}$ dose. Given the likely increased CL in critically ill patients with COVID-19, it is unlikely that this exposure would be achieved with a single $8 \mathrm{mg} / \mathrm{kg}$ dose. A systematic review and meta-analysis of long-term use in RA patients suggested a possible link with higher doses of tocilizumab $(8 \mathrm{mg} / \mathrm{kg})$ to secondary infections, which was less evident in patients receiving lower doses $(4 \mathrm{mg} / \mathrm{kg})$ [21, 27], but this observation requires further clinical correlation.

\subsection{Evaluation of Tocilizumab Population PK in Approved Indications}

Tocilizumab was initially approved by the US FDA Center for Drug Evaluation and Research (CDER) for the treatment of RA in 2010 and has since been approved in over 75 countries for RA and related conditions. The approved intravenous dose is $4 \mathrm{mg} / \mathrm{kg}$ every 4 weeks, which can be increased to $8 \mathrm{mg} / \mathrm{kg}$ based on clinical response [28]. Frey et al. [29] performed a population PK analysis using pooled data from four phase III clinical trials in RA, and identified a two-compartment structural model with parallel linear and non-linear (Michaelis-Menten) elimination. The nonlinear portion of tocilizumab CL following administration is concentration-dependent. Accordingly, the half-life varies based on the dose administered. The non-linear portion of $\mathrm{CL}$ is believed to reflect target-mediated drug disposition after binding to the IL-6R [14, 29]. Of note, the expression of IL- 6 and sIL-6R is likely altered by a variety of patientspecific factors, including increased receptor expression in critical illness [30]. In the initial population PK analysis submitted to the FDA, body surface area (BSA) was identified as a significant covariate of total CL, with CL predicted to increase non-linearly with BSA [21]. Because the relationship between tocilizumab exposure and body size is nonlinear, predicted tocilizumab exposures were not matched across the body weight distribution with weight-based dosing (e.g., $8 \mathrm{mg} / \mathrm{kg}$ ) [21]. Exposure with a fixed $560 \mathrm{mg}$ dose was predicted to be more consistent across the body weight range than a dose of $8 \mathrm{mg} / \mathrm{kg}$; however, fixed dosing results in slightly lower exposures at high body weight compared with more normal body weights. Ultimately, the weightbased dosing studied in tocilizumab clinical development was retained despite inadequate exposure matching across the weight range, due to the absence of a clear safety signal and a lower clinical response rate for high body weight RA patients, despite higher relative exposures [21].

Subsequently, a fixed-dose subcutaneous dosing regimen was studied and approved for RA in 2013. The approved subcutaneous dose was $162 \mathrm{mg}$ every 1-2 weeks, with weekly dosing recommended initially in patients weighing $\geq 100 \mathrm{~kg}$ [31]. A second population PK model was 
developed using data from two phase III trials wherein both intravenous and subcutaneous dosing were evaluated [31]. This model was identical in terms of compartmental structural and parallel linear and non-linear elimination with the exception that an absorption parameter $\left(k_{\mathrm{a}}\right)$ was added to accommodate for the subcutaneous route [31]. In contrast to the first model, this population PK model scaled all CL (e.g., systemic and intercompartmental) and volume (e.g., central and peripheral) parameters to total body weight. Given the marginal decrease in exposures (higher CL) observed in patients with higher body weights, a weekly subcutaneous fixed dose (rather than every 2 weeks) was selected for this body-weight subgroup. Saturation of non-linear CL was observed with an $8 \mathrm{mg} / \mathrm{kg}$ intravenous dose administered every 4 weeks and with a fixed dose of $162 \mathrm{mg}$ subcutaneously administered every week, suggesting that fixed dosing is possible, as long as concentrations remain above the saturation threshold for non-linear CL [31].

Tocilizumab was subsequently approved for polyarticular juvenile idiopathic arthritis (PJIA) and systemic juvenile idiopathic arthritis (SJIA). The approved doses of intravenous tocilizumab are $10-12 \mathrm{mg} / \mathrm{kg}(<30 \mathrm{~kg})$ and $8 \mathrm{mg} /$ $\mathrm{kg}(\geq 30 \mathrm{~kg})$ for these indications, respectively [28]. The population PK model supporting the dose selection for these pediatric patients was structurally identical to the initial RA model; however, height and age were included as additional covariates on the central distribution volume [32]. Similar to the initial intravenous tocilizumab population PK model, BSA was identified as a covariate on CL, with height and age identified as covariates of central volume [32]. The 8 $\mathrm{mg} / \mathrm{kg}$ regimen was evaluated in the registrational study for pediatric patients with SJIA; however, it was noted that low body weight $(<30 \mathrm{~kg}$ ) pediatric patients had lower clinical response [26, 32]. Modeling was used to predict individual exposures with higher dosing $(12 \mathrm{mg} / \mathrm{kg}$ ) for the low body weight patients and demonstrated good concordance with exposures achieved in the higher body weight range. However, based on these limited data and a lack of disease similarity, it is unclear whether these doses would extrapolate to pediatric patients with COVID-19.

The most recent tocilizumab indication, which may exhibit the greatest similarity to COVID-19, is for the treatment of severe or life-threatening CRS secondary to CAR $\mathrm{T}$ therapy $[26,28]$. FDA approval for this indication was based on retrospective analysis of 45 adult and pediatric patients receiving tocilizumab for CRS in CAR T clinical trials [26]. Patients received either $12 \mathrm{mg} / \mathrm{kg}(<30 \mathrm{~kg})$ or 8 $\mathrm{mg} / \mathrm{kg}(\geq 30 \mathrm{~kg})$ as a single intravenous dose, which could be repeated. The key clinical pharmacology question in the FDA review of this CRS indication was the adequacy of the dosing regimen [26, 33]. Exposure-response analysis was not possible as only a single dose-level was evaluated and only sparse PK data were available. Accordingly, the FDA utilized the previously developed pediatric model to support dosing in SJIA and re-estimated the linear portion of systemic CL and central volume of distribution $\left(V_{\mathrm{c}}\right)$. All other PK parameters and interindividual variation estimates were fixed at the previously determined values [26]. The refined PK model required higher values for linear systemic CL $(0.5 \mathrm{~L} /$ day $)$ and the central distribution volume $(1.8 \mathrm{~L})$ to accurately predict the PK profiles of patients with severe or life-threatening CRS [26]. In the absence of defined exposure-response for either efficacy or safety, the target exposure was defined based on the highest observed $C_{\max }(649$ $\mathrm{mg} / \mathrm{L}$ ) with a single $28 \mathrm{mg} / \mathrm{kg}$ dose in five healthy subjects.

\subsection{Tocilizumab PK in Patients with Coronavirus Disease 2019 (COVID-19)}

Tocilizumab PK data in patients with COVID-19 are currently limited to a single population PK analysis [23]. Moes et al., conducted an open-label, single-center observational PK study of tocilizumab in 29 patients with severe COVID-19 who all received a dose of $8 \mathrm{mg} / \mathrm{kg}$ to a maximum of $800 \mathrm{mg}$. They collected 139 leftover laboratory samples for secondary use over a 20-day window, which were assayed to determine free tocilizumab and sIL-6R concentrations. CRP and clinical outcomes were also assessed. The final population model consisted of a single disposition compartment model with parallel first-order and non-linear CL. No significant covariate effects were identified. The estimates for $\mathrm{CL}$ and volume of the central compartment $\left(V_{\mathrm{c}}\right)$ were $0.725 \mathrm{~L} /$ day and 4.34 $\mathrm{L}$, respectively. The linear CL estimate was higher than the estimate in adult RA patients ( $0.2-0.3 \mathrm{~L} /$ day), pediatric SJIA patients (0.17), and pediatric and adult patients with CAR T-induced CRS (0.5 L/day). Central volume was consistent with adult RA patients $(4.5 \mathrm{~L})$ but larger than pediatric SJIA patients $(0.94 \mathrm{~L})$ and pediatric and adult patients with CRS (1.8 L); however comparisons across disease states are limited by variation in the demographic characteristics (age, body size, etc.) of the underlying populations.

Simulations were performed to evaluate weight-based and fixed-dosing regimens with the conclusion that a 600 mg fixed dose may improve exposure matching across body weight; however, the limited sample precluded robust assessment of covariate effects (e.g., body size), which limits the external validity of conclusions about exposure variation with body weight. It is noteworthy that $\mathrm{CL}$ was high in this cohort and most consistent with pediatric and adult patients with CRS; however, interindividual variation in CL and volume was modest and more consistent with patients with stable inflammatory diseases. 


\subsection{How do Interleukin (IL)-6 and IL-6 Receptor Expression Compare Between COVID-19 and Other Inflammatory Conditions?}

When extrapolating dosing from one disease state to another, a critical consideration is whether or not disease similarity exists between the conditions. IL- 6 belongs to the glycoprotein 130 (gp130)-related family of cytokines, responsible for host inflammatory regulation as well as cell growth and differentiation [34]. Notably, IL-6 is distinct from other proinflammatory cytokines due to the natural soluble form of the IL-6R (i.e., sIL-6R) which is detectable in human serum during periods of inflammation or infection, in addition to the membrane-bound IL-6R expressed on specific cells [35]. The majority of soluble cytokine receptors are associated with antagonistic functions, while the sIL-6R serves as an agonist function and facilitates cytokine signaling $[35,36]$. Both membrane-bound IL-6R and sIL-6R are capable of participating in IL-6-mediated cell signaling, referred to as classical and trans-signaling, respectively [24, 25, 35-37]. Trans-signaling may cause cells without a membrane-bound IL-6R, including epithelial cells, smooth muscle cells, and endothelial cells, to have a proinflammatory response to IL-6 in critical illness [25]. Serum concentrations of IL-6 are elevated in chronic immune-mediated inflammatory diseases and have been shown to correlate with chronic disease activity, with IL-6 expression varying by disease condition [24]. IL-6 concentrations in patients with COVID-19 are heterogeneous [38-40] but generally similar to concentrations observed in patients with RA. COVID-19 patients with higher IL-6 concentrations have more severe disease and an increased risk of mortality [30, 38, 40, 41]. This trend mirrors observations from critically ill patients with severe sepsis, where non-survivors had higher mean serum IL-6 levels compared with survivors [42]. Of note, IL-6 levels in patients with acute respiratory distress syndrome (ARDS) and septic shock are variable and depend on the sampling method and observation time point within the course of illness (i.e., peaking early with acute inflammation, and then rapidly decreasing to undetectable levels) [25, 43, 44]. In contrast, IL-6 concentrations are significantly lower in patients with COVID-19 compared with patients with CRS associated with CAR T-cell therapy [13, 39, 43, 45]. Critically ill patients with COVID-19 generally present with elevated inflammatory markers (e.g., IL-6, sIL-6R, CRP) compared with those with mild to moderate illness [40, 43-45]. Single-cell RNA-sequencing (RNA-seq) analysis of peripheral blood failed to identify circulating cells as the source of IL-6 in COVID-19 [46, 47], which supports the hypothesis that cells within the alveolar space rather than the periphery may be the source of enhanced IL- 6 expression in COVID-19. A prospective observational trial compared IL-6 expression within the lung of patients with COVID-19 pneumonia and patients with pneumonia not caused by COVID-19 [48]. Bulk RNA-seq analysis of flow cytometrysorted alveolar macrophages, collected using bronchoalveolar lavage, revealed that IL-6 expression was not different between patients with COVID-19 pneumonia compared with patients with pneumonia due to other pathogens [48].

Although IL-6 concentrations have historically been used as a surrogate for clinical response in chronic inflammatory conditions, recent evidence suggests that sIL-6R may be a more informative marker of immune dysregulation and a better predictor of tocilizumab response [30, 49]. Correspondingly, sIL-6R may also be a better surrogate for clinical response with tocilizumab in COVID-19. The proposed rationale for evaluating sIL-6R response is that transsignaling via sIL-6R may provide a more complete picture of clinically relevant IL-6 activity in vivo [49]. Nishimoto conducted a tocilizumab PK/PD study in patients with RA and Castleman Disease and found that "as long as free tocilizumab was detectable, sIL-6R was saturated and IL-6 signaling was completely inhibited" [24]. The threshold concentration for saturation was $1 \mu \mathrm{g} / \mathrm{mL}$. However, even if sIL-6R is more predictive of tocilizumab anti-inflammatory response, it is much more challenging to measure clinically due to limited assay availability and accuracy [50].

Understanding sIL-6R response in COVID-19 is of interest given the observations in other inflammatory conditions. Unfortunately, data comparing sIL-6R expression with COVID-19 severity and response are limited. Koutsakos et al. [30] found that both IL-6 and sIL-6R concentrations were higher in critically ill patients with COVID-19 compared with patients who were not critically ill. They found that sIL-6R was marginally more accurate than IL-6 at predicting ward versus intensive care unit (ICU) admission status (area under the receiver operating characteristic curve [AUROC] 0.77 vs. 0.7) [40]. In the acute phase of COVID-19, both markers were elevated and exhibited a high degree of within-subject variability compared with healthy patients and those with convalescent disease [30]. Likewise, Moes et al. [23] measured sIL-6R response after tocilizumab administration and noted a rapid increase with a slow decline over 17 days. Based on their population PK model, they estimated that a threshold concentration of tocilizumab $5 \mu \mathrm{g} / \mathrm{mL}$ would be required to saturate sIL-6R (a value approximately fivefold higher than in non-COVID-19 patients). However, all patients maintained concentrations above this threshold for over 2 weeks and no difference in tocilizumab PK exposure was discerned between clinical response groups. As a result, uncertainty remains regarding whether sIL-6R monitoring would be useful in evaluating the adequacy of tocilizumab in patients with COVID-19.

Based on available evidence, it appears that IL- 6 expression in COVID-19 is more similar to that seen in patients with RA versus CRS; that lower IL-6 levels correspond 
with better clinical outcomes in COVID-19; and that IL-6 expression within the lung is similar between patients with COVID-19 and pneumonia due to other causes. Assuming IL-6 expression is an accurate marker of disease severity in COVID-19, one could argue that disease similarity exists between COVID-19 and other inflammatory conditions. However, IL-6 is an imperfect marker of clinical response in conditions such as RA [30]. Meanwhile, sIL-6R appears to potentially be a more precise measure of host inflammation versus IL-6 [30, 49]. Additionally, the pathophysiology driving IL-6 expression in COVID-19 is markedly different (acute viral infection) from conditions such as RA, PJIA, and SJIA (chronic autoimmune inflammation), and the relationship between tocilizumab treatment and occupancy of its target, IL-6R, is unknown in COVID-19. Therefore, extreme caution is warranted when extrapolating IL-6 and sIL-6R response to COVID-19. Finally, it remains unclear if a causal link exists between IL-6 expression and clinical response in COVID-19.

\subsection{Comparison of Fixed and Weight-Based Dosing Using Model-Informed Simulations}

To evaluate the extent to which a fixed dosing approach would yield exposures similar to those experienced by patients enrolled in COVID-19 clinical trials, we conducted focused model-informed dosing simulations. We utilized the distribution of body weight data from patients enrolled in the REMAPCAP trial to derive a reference range of $\mathrm{PK}$ exposures $[3,51]$. The model structure and parameter estimates were taken from the RA population PK model including both subcutaneous and intravenous administration [31], with linear CL increased to the value estimated in patient populations with CRS following CAR T, and used to simulate 100 trials with a sample size equal to the tocilizumab arm of REMAP-CAP $(n=353)$ [51] using NONMEM (version 7.4). Body weights were sampled from a random normal distribution (mean $90.3 \mathrm{~kg}$, SD $17.1 \mathrm{~kg}$ ) to reflect the median (interquartile range [IQR]) of patients enrolled in the tocilizumab arm of the trial [52].

From the 100 simulated trials, the median (5th, 95th percentiles) of individual exposure measures (i.e., AUC and day 7 minimum concentration $\left.\left[C_{\min }\right]\right)$ were calculated at the trial level, and the median value of each of these summary statistics across the 100 trials was plotted to define the reference exposure (blue shaded range) in Fig. 1. In a separate set of dose simulations, one patient per kilogram of body weight between 41 and $160 \mathrm{~kg}$ was generated and administered either a fixed or weight-banded dose, as well as a weight-based $8 \mathrm{mg} / \mathrm{kg}$ dose. A total of 1000 iterations were performed, resulting in 1000 simulated patients at each kilogram of body weight. Individual simulated exposure measures were calculated for each dosing condition for individual simulated patients, and the median and IQR exposure measures were calculated by binning simulated patients into body weight groups.
Figure 1a and $\mathrm{b}$ demonstrate that a fixed $400 \mathrm{mg}$ dose results in tocilizumab exposures below those likely achieved in REMAP-CAP, where an $8 \mathrm{mg} / \mathrm{kg}$ dose was used. A 400 $\mathrm{mg}$ fixed dose is predicted to achieve exposures below the 5 th percentile of predicted exposures from REMAP-CAP $(8 \mathrm{mg} / \mathrm{kg})$ in excess of $50 \%$ of patients weighing more than $66 \mathrm{~kg}$. Increasing intraindividual variation in the model (by a factor of four) resulted in a wider reference range (Fig. 1c and d) and marginally extended the weight range captured by $400 \mathrm{mg}$ fixed dosing. Day 14 trough concentrations were below $5 \mu \mathrm{g} / \mathrm{mL}$ for all but the lowest body weight group with a $400 \mathrm{mg}$ fixed dose. A higher $600 \mathrm{mg}$ fixed dose (Fig. 1e and $\mathrm{f}$ ) results in a greater proportion of simulated patients achieving exposure within the reference range but is still predicted to result in lower than reference exposure in the highest weight stratum, including a median predicted trough concentration $<5 \mu \mathrm{g} / \mathrm{mL}$ in this weight group.

Notably, across all of these simulation conditions, the 8 $\mathrm{mg} / \mathrm{kg}$ dosing arm is predicted to result in increasing exposure with increasing body weight, such that exposure is not matched across body weight groups. A weight-banded dosing approach with less frequent dose adjustments than RECOVERY, and using practical $200 \mathrm{mg}$ dosing increments, is predicted to result in improved exposure matching across the body weight range while still achieving exposures within the reference range predicted for REMAP-CAP (Fig. 1g and h). Median day 14 trough concentrations with this dosing approach were $>5 \mu \mathrm{g} / \mathrm{mL}$ with the entire IQR above this threshold for all but the smallest body weight groups.

\section{Discussion}

The optimal tocilizumab exposure in patients with COVID19 is not known. We sought to identify a fixed dosing regimen that is predicted to achieve exposures similar to the weight-based dosing regimens used in REMAP-CAP with the aim of informing drug conservation strategies [3]. We were not able to predict exposures from RECOVERY because patient body weight distributions were not provided in that trial [4]. Our simulations suggest that patients weighing $\geq 90 \mathrm{~kg}$ are unlikely to achieve exposures that fall within the reference range based on the predicted exposures within REMAP-CAP, even if a $600 \mathrm{mg}$ fixed dose was applied. Additionally, our analysis found that a weightbanded approach, similar to the dosing strategy employed in RECOVERY but with more conservative dose adjustments (using practical $200 \mathrm{mg}$ increments and alternate weight bands), yielded exposures that fell within the $90 \%$ predictive interval of exposures from REMAP-CAP. Our findings support exploration of an alternate weight-banded dosing strategy, but this dosing strategy will require clinical validation. We look forward to further data to inform tocilizumab 

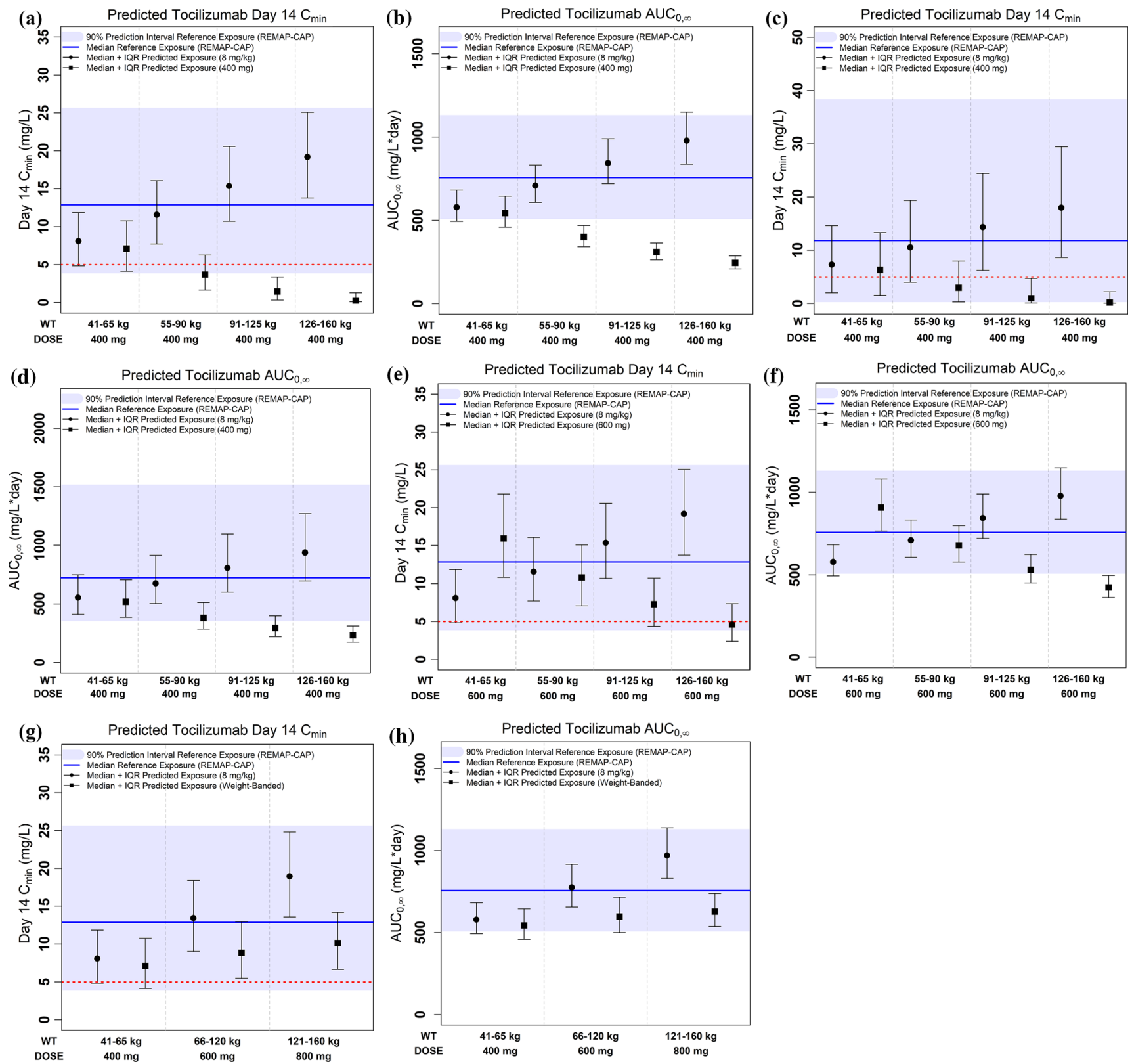

Fig. 1 a Predicted $C_{\min }$ for $400 \mathrm{mg}$ and $8 \mathrm{mg} / \mathrm{kg}$ dosing using the reference model. b Predicted $\mathrm{AUC}_{\infty}$ for $400 \mathrm{mg}$ and $8 \mathrm{mg} / \mathrm{kg}$ dosing using the reference model. c Predicted $C_{\min }$ for $400 \mathrm{mg}$ and $8 \mathrm{mg} / \mathrm{kg}$ dosing using the reference model with fourfold inflation of interindividual variability. d Predicted $\mathrm{AUC}_{\infty}$ for $400 \mathrm{mg}$ and $8 \mathrm{mg} / \mathrm{kg}$ dosing using the reference model with fourfold inflation of interindividual variability. e Predicted $C_{\min }$ for $600 \mathrm{mg}$ and $8 \mathrm{mg} / \mathrm{kg}$ dosing using the

PK/PD in COVID-19 disease, such as from the COVACTA study (which is planning to collect serum concentrations of IL-6, sIL-6R, and CRP with weight-based doses of tocilizumab $8 \mathrm{mg} / \mathrm{kg}$ ) [53].

On the other hand, our simulation results may not fully generalize to critically ill COVID-19 patients. It remains unclear whether the tocilizumab exposures approximated reference model. f Predicted $\mathrm{AUC}_{\infty}$ for $600 \mathrm{mg}$ and $8 \mathrm{mg} / \mathrm{kg}$ dosing using the reference model. $g$ Predicted $C_{\min }$ for weight-banded fixed dosing and $8 \mathrm{mg} / \mathrm{kg}$ dosing using the reference model. h Predicted $\mathrm{AUC}_{\infty}$ for weight-banded fixed dosing and $8 \mathrm{mg} / \mathrm{kg}$ dosing using the reference model. $C_{\min }$ minimum concentration, $A U C_{\infty}$ area under the concentration-time curve from time zero to infinity, $I Q R$ interquartile range, $W T$ weight

from the large COVID-19 platform trials are necessary, given the relatively low drug concentrations required to saturate the sIL-6R signaling pathway-a pathway that appears potentially more predictive of clinical outcomes than IL-6 alone. It is known that sIL-6R levels are significantly higher in critically ill COVID-19 patients compared with non-critically ill COVID-19 patients and healthy 
volunteers, which may lead to more rapid target-mediated tocilizumab CL [30]. Furthermore, protein and immunoglobulin (Ig) G catabolism has been shown to be increased in critically ill patients in general. This may partly explain the lower exposures seen in patients with CAR T therapyassociated CRS compared with patients with PJIA [43]. Systemic CL and $V_{\mathrm{d}}$ of tocilizumab are higher in critically ill patients with severe or life-threatening CRS than patients with COVID-19 [23]. Of note, albumin and total protein were also modifiers of tocilizumab $\mathrm{CL}$ in the final tocilizumab PK model [21]. Additionally, corticosteroids, which are now the standard of care in all COVID-19 patients who require supplemental oxygen, are associated with upregulation of IL-6R (including sIL6-R) [54, 55]. Increased target expression could again contribute to enhanced target-mediated $C L$ and could reduce concentrations below saturation levels sooner. Interestingly, although IL-6 is generally not elevated during pregnancy, sIL-6R concentrations are higher in pregnant women compared with non-pregnant women [56]. The increase in blood volume associated with pregnancy may further reduce tocilizumab concentrations, necessitating larger doses; additional studies are needed in pregnant patients. Although fixed doses appear to produce more homogenous PK exposures in a small number of patients with COVID-19 [23], there is likely to be higher between-patient variability in drug exposure among critically ill patients. Of note, our simulations and those performed by Moes et al. both showed increasing exposure with increasing body weight; however, it is unclear whether the resulting drug exposure is necessary for clinical efficacy or affects toxicity. Thus, more work is needed to understand the clinical PK and PK/PD of tocilizumab in COVID-19.

Higher doses of intravenous tocilizumab chosen for RA allow for extended interval dosing in the outpatient setting (i.e., every 4 weeks), which may not be necessary in an acute condition such as COVID-19. Given that sIL-6R saturation appears to be achieved with relatively low tocilizumab concentrations $(1-5 \mu \mathrm{g} / \mathrm{mL})[23,24]$, it is possible that lower doses could be as effective as higher doses for the treatment of COVID-19 [13, 21]. Limited observational data suggest that the therapeutic effect of tocilizumab in COVID-19 is not compromised when using lower doses (40-200 mg) [57] or a fixed dose of tocilizumab $(400 \mathrm{mg})[11,58]$. However, randomized controlled trials have not yet evaluated this approach. Additionally, it has been suggested that the relatively lower IL-6 levels in COVID-19, compared with CRS, may potentially support consideration of lower IL-6R antagonist doses in response to lower cytokine concentrations [13]. A lower tocilizumab dose that achieves receptor saturation, but for a shorter duration, could also theoretically minimize the risk of secondary opportunistic infections associated with prolonged IL-6 blockade without compromising efficacy.
Finally, due to the lack of objective targets, the adaptive trials conducted to date have allowed redosing of tocilizumab at the discretion of treating clinicians, largely based on clinical response [3,4]. Given the expected half-life of tocilizumab ranging from 11 to 13 days, additional doses of this therapy based on clinical response are likely unnecessary in COVID-19; avoiding second doses could further reserve its limited supply $[9,10,28]$.

\section{Conclusion}

In the treatment of COVID-19, it is currently unclear whether weight-based dosing provides advantages over a fixed-dosing strategy. The largest published trials to date establishing the benefit of tocilizumab in COVID-19 have used weight-based dosing and/or dose-banding strategies. In our study, fixed tocilizumab dosing of 400 and $600 \mathrm{mg}$ across each body weight strata did not consistently approximate the tocilizumab exposures (i.e., AUC) estimated from the REMAP-CAP trial showing benefit. Currently, a dose-exposure-response relationship for tocilizumab in the treatment of COVID-19 is not yet described and there remains uncertainty regarding the extent to which COVID19 may alter individual PK parameters. The use of a fixed dosing regimen may reduce the probability of 'underdosing' in some patients, whereas the risk of 'overexposure' with weight-based dosing in high-weight patients is nontrivial. To date, the clinical contribution of 'overexposure' on the risk of secondary infections is unclear, although higher doses have been used without affecting neutrophil counts [26]. Since an established PK/PD relationship for tocilizumab is not known, it is reasonable to use an exposure matching approach in an effort to target exposures that have demonstrated efficacy in the target population [14]. Our simulations found that, in contrast to fixed dosing, an alternate 'weight-banded' dosing approach produced the best approximation of the REMAP-CAP population exposures. The real-world impact on outcomes of a fixed-dose approach in the setting of critical limitations in drug supply, in an attempt to treat as many patients as possible who would qualify for tocilizumab therapy, is yet to be determined.

We recommend that if jurisdictions or individual hospital sites move to adopt alternate dosing approaches (a likely possibility in the scenario where ethical rationing of limited drug product is in effect), it is studied (ideally with real-time monitoring) to provide data on patient-centered outcomes and the real-world effect of a fixed dose, or lowerdose tocilizumab therapy, in COVID-19 disease. In addition, the current landscape of clinical trials is rapidly evolving and adaptive trials have proven especially useful during this pandemic due to their agile and flexible design in exploring 
repurposed therapies. Leveraging platform trial design to identify optimal dosing strategies, also known as 'socially optimal' dosing, will additionally enable the overall realization of maximum clinical benefits while mitigating inequities that may arise from increasing global demand and local/ regional limitations in drug supply [59]. We highlight here the importance of rational dosing of medications through $\mathrm{PK} / \mathrm{PD}$, and strongly advocate that current and future trials incorporate PK/PD considerations into their trial designs in order to better inform optimized dosing strategies at the bedside.

Acknowledgements The authors would like to acknowledge Dr. Srinivas Murthy (University of British Columbia) and the REMAP-CAP trial team for providing us with the weight distributions of the study population in the REMAP-CAP study for our PK modeling analysis.

\section{Declarations}

Funding No institutional or corporate funding was received by any of the authors for this work.

Conflict of interest Elizabeth Leung, Ryan L. Crass, Sarah C.J. Jorgensen, Sumit Raybardhan, Bradley J. Langford, W. Justin Moore, and Nathaniel J. Rhodes declare no conflicts of interest, including pertinent commercial and other relationships, related to this work. Sarah C.J. Jorgensen has previously received a presenter's honorarium from $\mathrm{Su}-$ novion, unrelated to the present work. Elizabeth Leung has previously received a presenter's honorarium from the Ontario Pharmacists' Association, unrelated to the present work. Nathaniel J. Rhodes reports honoraria from ASHP and receipt of research funds from AACP and Paratek, unrelated to the present work. Sumit Raybardhan has previously received a presenter's honorarium from Merck, unrelated to the present work.

Availability of Data and Material Data sharing was not applicable to this article as no datasets were generated or analyzed during the current work (this is a review article, not a primary study).

Code Availability Not applicable (this is a review article, not a primary study).

\section{Modeling Software NONMEM (version 7.4).}

Plotting Software R (version 3.6.3).

Author Contributions EL wrote the first draft of the manuscript. EL, $\mathrm{SR}$, and BL contributed to the conception of the manuscript. NJR and RLC provided methodology support and performed PK/PD modeling. All authors (EL, SJ, SR, BL, RLC, WJM, NJR) revised the manuscript critically for important intellectual content and approved the final version.

Ethics Approval/Consent to Participate/Consent for Publication Not applicable (review article).

\section{References}

1. Infectious Diseases Society of America. ISDA Guidelines on the Treatment and Management of Patients with COVID-19. Version 4.2.0. 2021. https://www.idsociety.org/practice-guideline/covid19-guideline-treatment-and-management/. Accessed 7 May 2021.

2. National Institutes of Health. COVID-19 Treatment Guidelines Panel. Coronavirus Disease 2019 (COVID-19) Treatment Guidelines. 2021. https://www.covid19treatmentguidelines.nih.gov/. Accessed 7 May 2021.

3. Gordon AC, Mouncey PR, Al-Beidh F, and the REMAP-CAP Investigators. Interleukin- 6 receptor antagonists in critically ill patients with Covid-19. N Engl J Med. 2021;384(16):1491-1502. https://doi.org/10.1056/NEJMoa2100433.

4. Horby P, Lim WS, Emberson JR; and the RECOVERY Collaborative Group. Tocilizumab in patients admitted to hospital with COVID-19 (RECOVERY): a randomised, controlled, open-label, platform trial. Lancet. 2021;397(10285):1637-45.

5. Kewan T, Covut F, Al-Jaghbeer MJ, Rose L, Gopalakrishna $\mathrm{KV}$, Akbik B. Tocilizumab for treatment of patients with severe COVID-19: a retrospective cohort study. EClinicalMedicine. 2020;24: 100418. https://doi.org/10.1016/j.eclinm.2020.100418.

6. Vu CA, DeRonde KJ, Vega AD, Maxam M, Holt G, Natori Y, et al. Effects of tocilizumab in COVID-19 patients: a cohort study. BMC Infect Dis. 2020;20(1):964. https://doi.org/10.1186/ s12879-020-05701-4.

7. Gokhale Y, Mehta R, Kulkarni U, Karnik N, Gokhale S, Sundar U, et al. Tocilizumab improves survival in severe COVID-19 pneumonia with persistent hypoxia: a retrospective cohort study with follow-up from Mumbai, India. BMC Infect Dis. 2021;21(1):241. https://doi.org/10.1186/s12879-021-05912-3.

8. Murthy S, Lee TC. IL-6 blockade for COVID-19: a global scientific call to arms. Lancet Respir Med. 2021;9(5):438-40. https:// doi.org/10.1016/S2213-2600(21)00127-2.

9. Morris AM, et al. Strategies to manage tocilizumab supply during the COVID-19 Pandemic. Ontario COVID-19 Science Advisory Table. 2021. https://doi.org/10.47326/ocsat.2021.02.22.1.0. Accessed 19 Apr 2021.

10. British Columbia Centre for Disease Control. Clinical guidanceantimicrobial and immunomodulatory therapy in adult patients with COVID-19. 2021. http://www.bccdc.ca/health-profession als/clinical-resources/covid-19-care/clinical-care/treatments. Accessed 18 Apr 2021

11. Guaraldi G, Meschiari M, Cozzi-Lepri A, Milic J, Tonelli R, Menozzi M, et al. Tocilizumab in patients with severe COVID-19: a retrospective cohort study. Lancet Rheumatol. 2020;2(8):e47484. https://doi.org/10.1016/S2665-9913(20)30173-9.

12. Dejaco C, Alunno A, Bijlsma JW, Boonen A, Combe B, Finckh A, et al. Influence of COVID-19 pandemic on decisions for the management of people with inflammatory rheumatic and musculoskeletal diseases: a survey among EULAR countries. Ann Rheum Dis. 2020;80:4. https://doi.org/10.1136/annrheumdis-2020-218697.

13. Yang C, Liu M. Tocilizumab in treatment for patients with COVID-19. JAMA Intern Med. 2021;181(7):1017-8. https://doi. org/10.1001/jamainternmed.2021.0392.

14. Mould DR, Green B. Pharmacokinetics and pharmacodynamics of monoclonal antibodies: concepts and lessons for drug development. BioDrugs. 2010;24(1):23-39. https://doi.org/10.2165/11530 560-000000000-00000. 
15. Feldschuh J, Enson Y. Prediction of the normal blood volume. Relation of blood volume to body habitus. Circulation. 1977;56:605-12.

16. Lemmens HJ, Bernstein DP, Brodsky JB. Estimating blood volume in obese and morbidly obese patients. Obes Surg. 2006;16:773-6.

17. Peters AM, Snelling HL, Glass DM, Love S, Bird NJ. Estimated lean body mass is more appropriate than body surface area for scaling glomerular filtration rate and extracellular fluid volume. Nephron Clin Pract. 2010;116(1):c75-80. https://doi.org/10.1159/ 000314666.

18. Pai MP. Drug dosing based on weight and body surface area: mathematical assumptions and limitations in obese adults. Pharmacotherapy. 2012;32(9):856-68. https://doi.org/10.1002/j.18759114.2012.01108.x.

19. Goyal R, Faizy AF, Siddiqui SS, Singhai M. Evaluation of TNF- $\alpha$ and IL-6 levels in obese and non-obese diabetics: pre- and postinsulin effects. N Am J Med Sci. 2012;4(4):180-4. https://doi.org/ 10.4103/1947-2714.94944.

20. Sindhu S, Thomas R, Shihab P, Sriraman D, Behbehani K, Ahmed $\mathrm{R}$. Obesity is a positive modulator of IL-6R and IL-6 expression in the subcutaneous adipose tissue: significance for metabolic inflammation. PLoS ONE. 2015;10(7): e0133494. https://doi. org/10.1371/journal.pone.0133494.

21. US FDA Center for Drug Evaluation and Research (CDER). Clinical Pharmacology and Biopharmaceutics Reviews. 2010. Tocilizumab BLA125276. 2021. https://www.accessdata.fda.gov/drugs atfda_docs/nda/2010/125276s000ClinPharmR.pdf. Accessed 13 Apr 2021.

22. Bai S, Jorga K, Xin Y, Jin D, Zheng Y, Damico-Beyer LA, et al. A guide to rational dosing of monoclonal antibodies. Clin Pharmacokinet. 2012;51(2):119-35.

23. Moes DJAR, van Westerloo DJ, Arend SM, Swen JJ, de Vries A, Guchelaar HJ, et al. Towards fixed dosing of tocilizumab in ICU-admitted COVID-19 patients: results of an observational population pharmacokinetic and descriptive pharmacodynamic study. Clin Pharmacokinet. 2021. https://doi.org/10.1007/ s40262-021-01074-2.

24. Nishimoto N, Terao K, Mima T, Nakahara H, Takagi N, Kakehi $\mathrm{T}$. Mechanisms and pathologic significances in increase in serum interleukin-6 (IL-6) and soluble IL-6 receptor after administration of an anti-IL-6 receptor antibody, tocilizumab, in patients with rheumatoid arthritis and Castleman disease. Blood. 2008;112(10):3959-64. https://doi.org/10.1182/ blood-2008-05-155846.

25. McElvaney OJ, Curley GF, Rose-John S, McElvaney NG. Interleukin-6: obstacles to targeting a complex cytokine in critical illness. Lancet Respir Med. 2021. https://doi.org/10.1016/S22132600(21)00103-X

26. US FDA Center for Drug Evaluation and Research (CDER). Multi-discipline Review. Tocilizumab 125276Orig1s114. 2021. https://www.accessdata.fda.gov/drugsatfda_docs/nda/2017/12527 6Orig1s114MultidisciplineR.pdf. Accessed 5 May 2021

27. Campbell L, Chen C, Bhagat SS, Parker RA, Östör AJK. Risk of adverse events including serious infections in rheumatoid arthritis patients treated with tocilizumab: a systematic literature review and meta-analysis of randomized controlled trials. Rheumatology. 2011;50(3):552-62. https://doi.org/10.1093/rheumatology/ keq343.

28. US FDA. Tocilizumab Prescribing Information. 2013. https:// www.accessdata.fda.gov/drugsatfda_docs/label/2013/125276s092 lbl.pdf. Accessed 13 Apr 2021.

29. Frey N, Grange S, Woodworth T. Population pharmacokinetic analysis of tocilizumab in patients with rheumatoid arthritis. J Clin Pharmacol. 2010;50(7):754-66. https://doi.org/10.1177/ 0091270009350623.
30. Koutsakos M, Rowntree LC, Hensen L, Chua BY, van de Sandt $\mathrm{CE}$, Habel JR, et al. Integrated immune dynamics define correlates of COVID-19 severity and antibody responses. Cell Rep Med. 2021;2(3):100208. https://doi.org/10.1016/j.xcrm.2021.100208.

31. US FDA Center for Drug Evaluation and Research (CDER). Clinical Pharmacology and Biopharmaceutics Reviews. Tocilizumab BLA125472Orig1s000. 2013. https://www.accessdata.fda.gov/ drugsatfda_docs/nda/2013/125472Orig1s000ClinPharmR.pdf. Accessed 5 May 2021.

32. US FDA Center for Drug Evaluation and Research (CDER). Clinical Pharmacology Review. Tocilizumab BLA125276, supplement 22. 2010. https://www.fda.gov/media/80466/download. Accessed 5 May 2021.

33. Le RQ, Li L, Yuan W, Shord SS, Nie L, Habtemariam BA, et al. FDA approval summary: tocilizumab for treatment of chimeric antigen receptor $\mathrm{T}$ cell-induced severe or life-threatening cytokine release syndrome. Oncologist. 2018;23(8):943-7. https://doi.org/ 10.1634/theoncologist.2018-0028.

34. Peters M, Jacobs S, Ehlers M, Vollmer P, Müllberg J, Wolf E, et al. The function of the soluble interleukin 6 (IL-6) receptor in vivo: sensitization of human soluble IL-6 receptor transgenic mice towards IL- 6 and prolongation of the plasma half-life of IL-6. J Exp Med. 1996;183(4):1399-406. https://doi.org/10.1084/ jem.183.4.1399.

35. Rose-John S. IL-6 trans-signaling via the soluble IL-6 receptor: importance for the pro-inflammatory activities of IL-6. Int J Biol Sci. 2012;8(9):1237-47. https://doi.org/10.7150/ijbs.498.

36. Jones SA, Rose-John S. The role of soluble receptors in cytokine biology: the agonistic properties of the sIL-6R/IL-6 complex. Biochim Biophys Acta. 2002;1592(3):251-63. https://doi.org/ 10.1016/s0167-4889(02)00319-1.

37. Jones SA, Scheller J, Rose-John S. Therapeutic strategies for the clinical blockade of IL-6/gp130 signaling. J Clin Invest. 2011;121(9):3375-83. https://doi.org/10.1172/JCI57158.

38. Zhou F, Yu T, Du R, Fan G, Liu Y, Liu Z, et al. Clinical course and risk factors for mortality of adult inpatients with COVID19 in Wuhan, China: a retrospective cohort study [published erratum appears in Lancet. 2020 Mar;395(10229):1038]. Lancet. 2020;395(10229):1054-62. https://doi.org/10.1016/S01406736(20)30566-3.

39. Leisman DE, Ronner L, Pinotti R, Taylor MD, Sinha P, Calfee CS, et al. Cytokine elevation in severe and critical COVID-19: a rapid systematic review, meta-analysis, and comparison with other inflammatory syndromes. Lancet Respir Med. 2020;8(12):123344. https://doi.org/10.1016/S2213-2600(20)30404-5.

40. Coomes EA, Haghbayan H. Interleukin-6 in Covid-19: a systematic review and meta-analysis. Rev Med Virol. 2020;30(6):1-9. https://doi.org/10.1002/rmv.2141.

41. Ruan Q, Yang K, Wang W, Jiang L, Song J. Clinical predictors of mortality due to COVID-19 based on an analysis of data of 150 patients from Wuhan, China [published erratum appears in Intensive Care Med. 2020;46(6):1294-1297]. Intensive Care Med. 2020;46(5):846-8. https://doi.org/10.1007/s00134-020-05991-x.

42. Zeni F, Tardy B, Vindimian M, Pain P, Gery P, Bertrand JC. Soluble interleukin-6 receptor in patients with severe sepsis. J Infect Dis. 1995;172(2):607-8. https://doi.org/10.1093/infdis/172.2.607.

43. Hedrick TL, Murray BP, Hagan RS, Mock JR. COVID-19: clean up on IL-6. Am J Respir Cell Mol Biol. 2020;63(4):541-3. https:// doi.org/10.1165/rcmb.2020-0277LE.

44. Del Valle DM, Kim-Schulze S, Huang HH, Beckmann ND, Nirenberg S, Wang B, et al. An inflammatory cytokine signature predicts COVID-19 severity and survival. Nat Med. 2020;26(10):1636-43.

45. Gao Y, Li T, Han M, Li X, Wu D, Xu Y, et al. Diagnostic utility of clinical laboratory data determinations for patients with the 
severe COVID-19. J Med Virol. 2020;92(7):791-6. https://doi. org/10.1002/jmv.25770.

46. Wilk AJ, Rustagi A, Zhao NQ, Roque J, Martínez-Colón GJ, McKechnie JL, et al. A single-cell atlas of the peripheral immune response in patients with severe COVID-19. Nat Med. 2020;26:1070-6.

47. Schulte-Schrepping J, Reusch N, Paclik D, Baßler K, Schlickeiser $S$, Zhang B. Deutsche COVID-19 OMICS Initiative (DeCOI). Severe COVID-19 is marked by a dysregulated myeloid cell compartment. Cell. 2020;182:1419-40.

48. Grant RA, Morales-Nebreda L, Markov NS, Swaminathan S, Querrey M, Guzman ER, NU SCRIPT Study Investigators, et al. Circuits between infected macrophages and T cells in SARSCoV-2 pneumonia. Nature. 2021;590(7847):635-41. https://doi. org/10.1038/s41586-020-03148-w.

49. Chen LYC, Biggs CM, Jamal S, Stukas S, Wellington CL, Sekhon MS. Soluble interleukin-6 receptor in the COVID-19 cytokine Storm syndrome. Cell Rep Med. 2021;2(5):100269. https://doi. org/10.1016/j.xcrm.2021.100269.

50. Chen F, Teachey DT, Pequignot E, Frey N, Porter D, Maude SL, et al. Measuring IL-6 and sIL-6R in serum from patients treated with tocilizumab and/or siltuximab following CAR T cell therapy. J Immunol Methods. 2016;434:1-8. https://doi.org/10.1016/j.jim. 2016.03.005.

51. Personal communication, author e-mail communication. Dr. Srinivas Murthy. 2021.

52. Wan X, Wang W, Liu J, Tong T. Estimating the sample mean and standard deviation from the sample size, median, range and/ or interquartile range. BMC Med Res Methodol. 2014;14:135. https://doi.org/10.1186/1471-2288-14-135.

53. ClinicalTrials.gov. Bethesda (MD): National Library of Medicine (US). ClinicalTrials.gov Identifier NCT04320615. A study to evaluate the safety and efficacy of tocilizumab in patients with severe COVID-19 Pneumonia (COVACTA). 2021. https://clini
caltrials.gov/ct2/show/NCT04320615https://clinicaltrials.gov/ct2/ show/NCT04320615. Accessed 1 May 2021.

54. Dovio A, Perazzolo L, Saba L, Termine A, Capobianco M, Bertolotto A, et al. High-dose glucocorticoids increase serum levels of soluble IL-6 receptor alpha and its ratio to soluble gp130: an additional mechanism for early increased bone resorption. Eur $\mathrm{J}$ Endocrinol. 2006;154(5):745-51. https://doi.org/10.1530/eje.1. 02147.

55. Rose-John S, Schooltink H, Lenz D, Hipp E, Dufhues G, Schmitz $\mathrm{H}$, et al. Studies on the structure and regulation of the human hepatic interleukin-6 receptor. Eur J Biochem. 1990;190(1):7983. https://doi.org/10.1111/j.1432-1033.1990.tb15548.x.

56. Matsuzaki N, Neki R, Sawai K, Shimoya K, Okada T, Sakata M, et al. Soluble interleukin-6 (IL-6) receptor in the sera of pregnant women forms a complex with IL-6 and augments human chorionic gonadotropin production by normal human trophoblasts through binding to the IL-6 signal transducer. J Clin Endocrinol Metab. 1995;80(10):2912-7. https://doi.org/10.1210/jcem.80.10.75598 74.

57. Strohbehn GW, Heiss BL, Rouhani SJ, Trujillo JA, Yu J, Kacew AJ, et al. COVIDOSE: a phase II clinical trial of low-dose tocilizumab in the treatment of noncritical COVID-19 pneumonia. Clin Pharmacol Ther. 2021;109(3):688-96. https://doi.org/10.1002/cpt. 2117.

58. Biran N, Ip A, Ahn J, Go RC, Wang S, Mathura S, et al. Tocilizumab among patients with COVID-19 in the intensive care unit: a multicentre observational study. Lancet Rheumatol. 2020;2(10):603-12. https://doi.org/10.1016/S2665-9913(20) 30277-0.

59. Strohbehn GW, Parker WF, Reid PD, Gellad WF. Socially optimal pandemic drug dosing. Lancet Glob Health. 2021;9(8):e1049-50. https://doi.org/10.1016/S2214-109X(21)00251-5. 